\title{
Editorial
}

\section{Experimental Shock and Vibration Analysis}

\author{
Roger Serra, ${ }^{1}$ Luigi Garibaldi, ${ }^{2}$ Nuno M. Maia, ${ }^{3}$ and Marc Thomas ${ }^{4}$ \\ ${ }^{1}$ INSA Centre Val de Loire, 3 rue de la chocolaterie, 41000 Blois, France \\ ${ }^{2}$ Politecnico di Torino, Corso Duca degli Abruzzi 24, 10129 Torino, Italy \\ ${ }^{3}$ LAETA, IDMEC, University of Lisbon, Instituto Superior Tecnico, Avenida Rovisco Pais, 1049-001 Lisboa, Portugal \\ ${ }^{4}$ École de Technologie Supérieure, 1100 Rue Notre-Dame Ouest, Montréal, Québec, Canada H3C 1 K3
}

Correspondence should be addressed to Roger Serra; roger.serra@insa-cvl.fr

Received 10 September 2015; Accepted 8 October 2015

Copyright (C) 2015 Roger Serra et al. This is an open access article distributed under the Creative Commons Attribution License, which permits unrestricted use, distribution, and reproduction in any medium, provided the original work is properly cited.

The analysis of shock and vibration responses is the starting point to investigate the dynamic properties of a system, a structure, or a mechanism, as well as its expected response to a given excitation (harmonic, random, shock, etc.). By this approach it can be determined whether a particular system, structure, or mechanism will fulfill its intended function; in addition, the results of the dynamic loadings acting on a structure can be predicted, such as dynamic stresses, fatigue life, and noise levels, so that its usefulness can be maintained and maximized. Furthermore, the analysis allows seeking those structural parameters most affecting the dynamic response so that if any improvement or change in the response is required, the structure can be modified in the most economic and appropriate way. The methods also made important great strides. Indeed, the vibratory analysis is not one simple complementary tool. It is the basis of many powerful techniques which make it possible, for example, to probe the structures or the health monitoring of materials during their service, to detect the defects and the damage, and to follow their evolution in real-time. The shock and vibration analyses are massively present today in the various branches of industry, from aeronautics to car manufacturing and from machining and maintenance to civil engineering, to mention a few areas, which have made this special issue a true need.

This scientific topic leading industrial and academic researchers' communities concerned by experimental shock and vibration analysis has highly advanced taking positive steps in recent years to develop more comprehensive and rational procedures for dynamic assessment. Starting from forty-eight submitted papers, only seventeen papers on modal analysis, vibration-based condition monitoring, damage detection and localization, experimental/numerical combined approach, physical experiments with advanced computational methods investigations, and their various engineering/industrial applications have been selected and included in this special issue after completing a careful and rigorous peer review process by the international scientific committee.

Although these papers do not make an exhaustive treatment of the entire experimental shock and vibration analysis topics, they reflect different interesting issues that have constituted important points of concern for researchers in this area. We trust that this document will contribute to disseminate the current trends and topics of interest in this fascinating and fast growing area and that it will be a valuable tool in research and professional activity.

Roger Serra

Luigi Garibaldi

Nuno M. Maia

Marc Thomas 

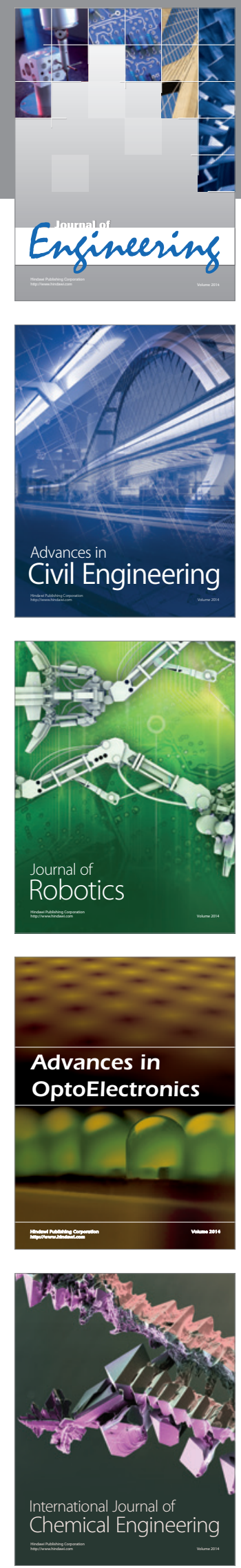

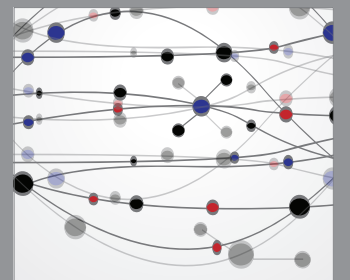

The Scientific World Journal
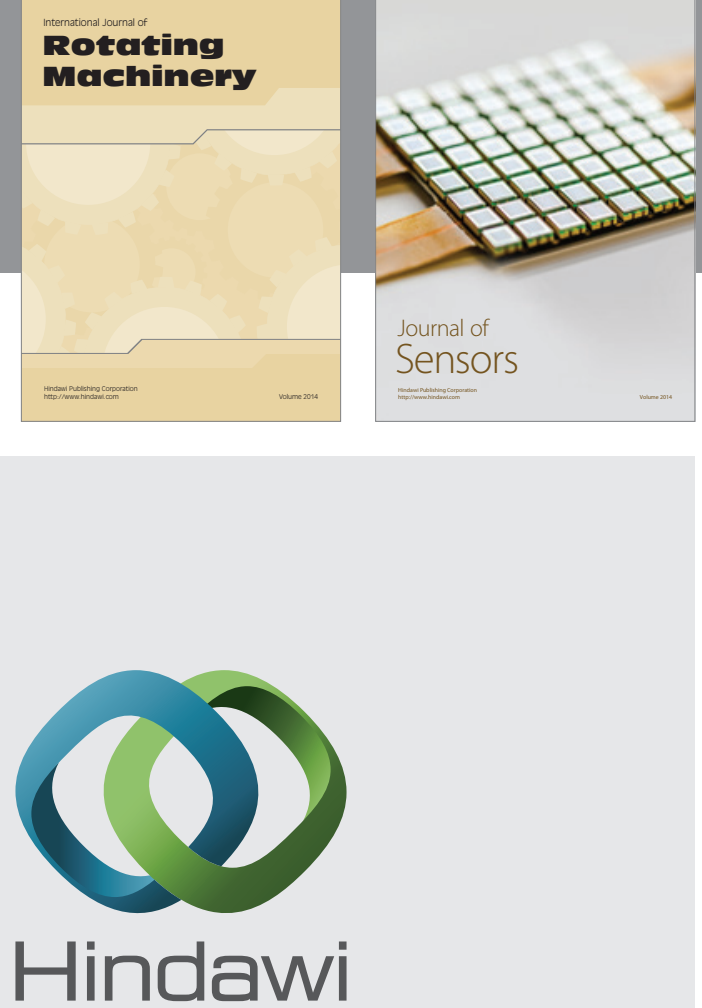

Submit your manuscripts at http://www.hindawi.com
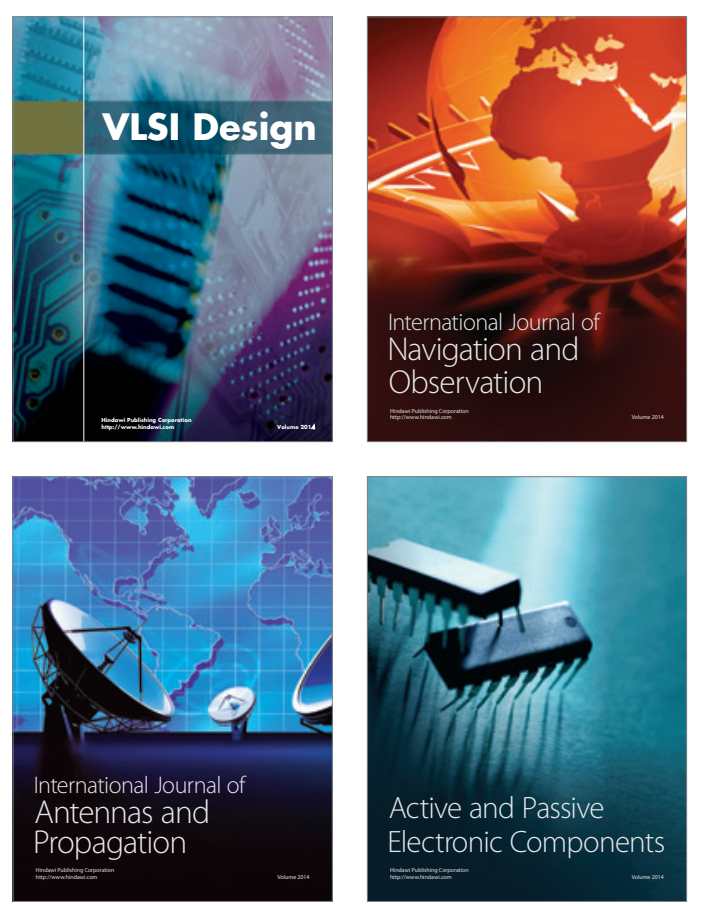
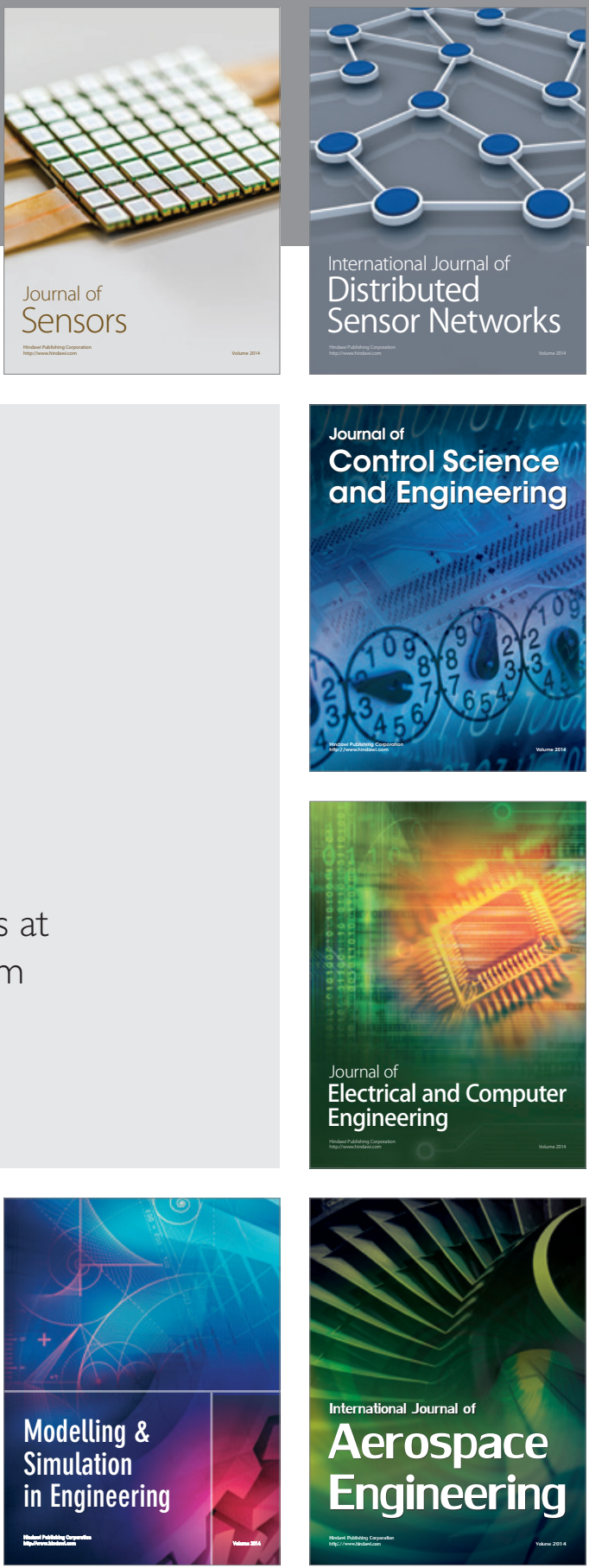

Journal of

Control Science

and Engineering
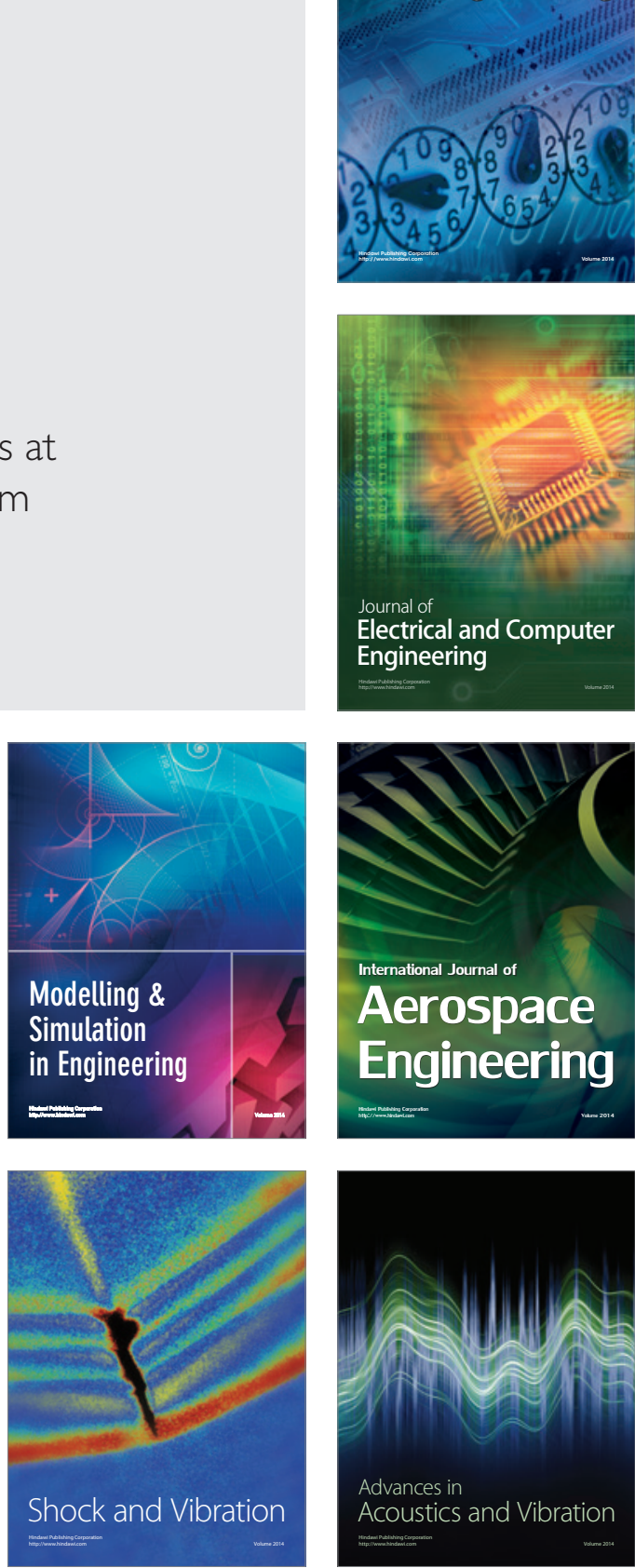